\title{
Bilateral Idiopathic Granulomatous Mastitis: A Case of Noncaseating Granulomas in a Patient With Latent Tuberculosis
}

\author{
Laura Malmut ${ }^{\mathrm{a}}$, George J. Tsioulias ${ }^{\mathrm{b}, \mathrm{c}}$
}

\begin{abstract}
Idiopathic granulomatous mastitis (IGM) is a rare benign inflammatory disease of the breast of unknown etiology. The typical clinical presentation is a unilateral palpable breast mass with pain and overlying skin erythema. IGM remains a diagnosis of exclusion, and it is necessary to exclude all infectious and noninfectious causes of granulomatous disease. Definitive diagnosis of IGM relies on histological examination, demonstrating granulomatous inflammation without caseous necrosis. Common medical treatment entails the use of corticosteroids and other immunosuppressive agents in patients with IGM. We present a rare case of bilateral IGM in a patient found to have latent tuberculosis (TB), and we discuss the challenges of treating IGM in a tuberculin skin test positive patient. The patient is a 44-year-old female veteran with a history of a positive tuberculin skin test who initially presented with a unilateral painful breast mass. She continued to present with recurrent painful breast masses that progressed to bilateral disease. Mammography and breast MRI were suspicious for malignancy and required biopsy. Histopathology revealed noncaseating granulomas. All stains and cultures were negative for mycobacterium. After 3 years, the disease subsided and she did not present with any further recurrences. She was subsequently treated with isoniazid to reduce the risk of TB reactivation. The diagnosis of IGM is challenging and should be considered in the differential diagnosis of persistent or recurrent breast masses. Patients with IGM found to have latent TB are of particular concern, and tuberculous mastitis must be carefully excluded. IGM is generally a self-limiting disease, and treatment options include observation, symptomatic palliation, steroids, and in complicated recurrent cases or cases involving an abscess, surgery may be considered as a last resort. Treatment of IGM in pa-
\end{abstract}

Manuscript accepted for publication July 4, 2014

${ }^{a}$ Rutgers New Jersey Medical School, 185 S Orange Ave, Newark, NJ 07103, USA

${ }^{\mathrm{b}}$ Department of General Surgery, U.S. Department of Veterans Affairs, 385 Tremont Ave. (112), East Orange, NJ 07018, USA

${ }^{\mathrm{c}}$ Corresponding Author: George J. Tsioulias, U.S. Department of

Veterans Affairs, 385 Tremont Ave. (112), East Orange, NJ 07018,

USA. Email: george.tsioulias@va.gov

doi: http://dx.doi.org/10.14740/jmc1835w tients with latent TB should be primarily symptomatic, and steroids should not be used.

Keywords: Granulomatous mastitis; Idiopathic; Tuberculosis; Infection; Steroid

\section{Introduction}

Granulomatous mastitis is a rare benign disease of the breast first described by Kessler and Wolloch in 1972 [1]. Idiopathic granulomatous mastitis (IGM) is an inflammatory disease of unknown etiology, but several factors have been associated including autoimmune and infectious processes. IGM usually affects women of childbearing age, and most have a recent history of breastfeeding. IGM additionally has been speculated to be associated with autoimmune disorders, oral contraceptive pills, hyperprolactinemia, fat necrosis, alpha1-antitrypsin deficiency and corynebacterium infection [24]. A proposed mechanism of the granulomatous inflammation is extravasation of protein and fat-rich secretions from damaged mammary ducts into the lobular connective tissue that causes a localized immune response $[4,5]$.

The typical clinical presentation is a unilateral palpable breast mass with pain and overlying skin erythema [6, 7]. Bilateral IGM is uncommon although it has been reported in literature [7]. The clinical findings of IGM are non-specific and can clinically and radiographically mimic inflammatory breast cancer [8]. IGM remains a diagnosis of exclusion, and it is necessary to exclude all infectious and non-infectious causes of granulomatous disease [9]. The differential diagnosis includes but is not limited to sarcoidosis, Wegener's granulomatosis, tuberculosis (TB) and fungal infection [8, 10]. Definitive diagnosis of IGM relies on histological examination, demonstrating granulomatous inflammation without caseous necrosis. Characteristic cytologic features include noncaseating granulomas, epithelioid histiocytes, multinucleated giant cells and a predominantly neutrophilic background $[9,11]$.

There is no standard treatment, but common methods use corticosteroids or other immunosuppressive agents with or without surgical excision [5]. 


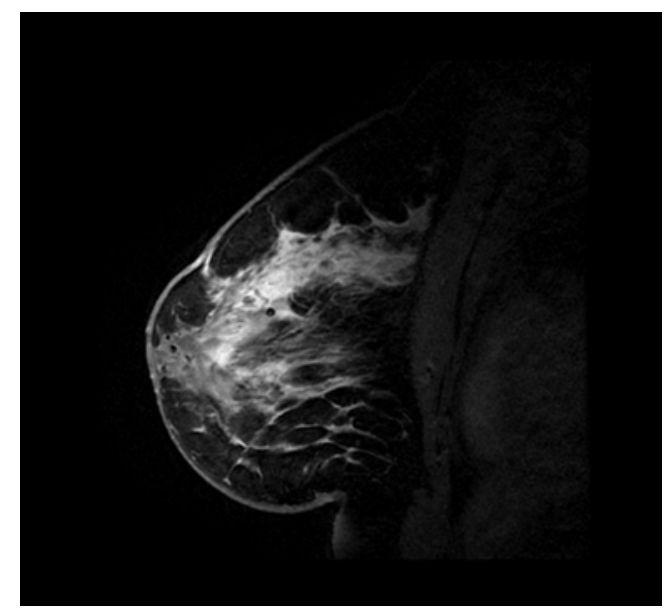

Figure 1. Contrast enhanced MRI sagittal view of the left breast demonstrating diffuse enhancement of the breast parenchyma.

In this report we describe a case of bilateral granulomatous mastitis in a PPD positive patient and discuss the implications and challenges of treating such a patient for IGM.

\section{Case Report}

The patient is a 44-year-old G4P4 Gulf War veteran who initially presented with a 3-day history of left breast pain. The breast was engorged with an area of induration in the 10 - 11 o'clock position, which was accompanied by tenderness and overlying erythema. She denied history of nipple discharge, oral contraceptive or estrogen use, breast trauma or family history of breast cancer. She reported breastfeeding all four of her children, with the last time being 14 years

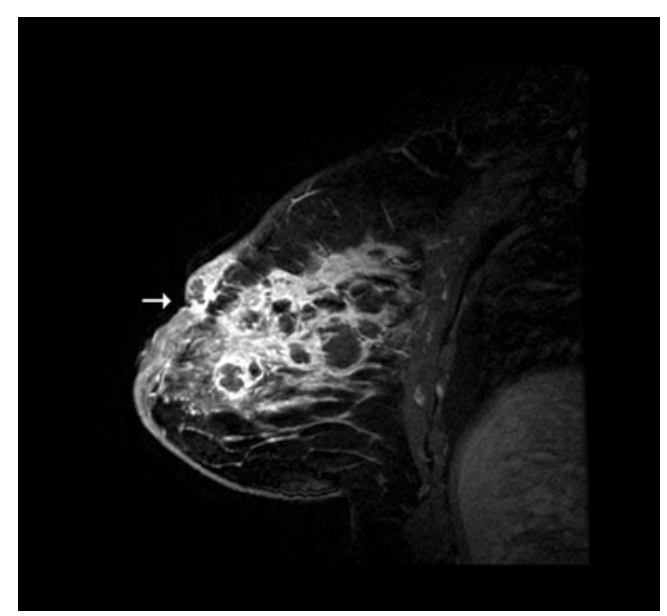

Figure 2. Contrast enhanced MRI sagittal view of the right breast showing solid and cystic changes with periareolar skin thickening, moderate nipple retraction and cystic lesion close to the skin representing a fistula (arrow).

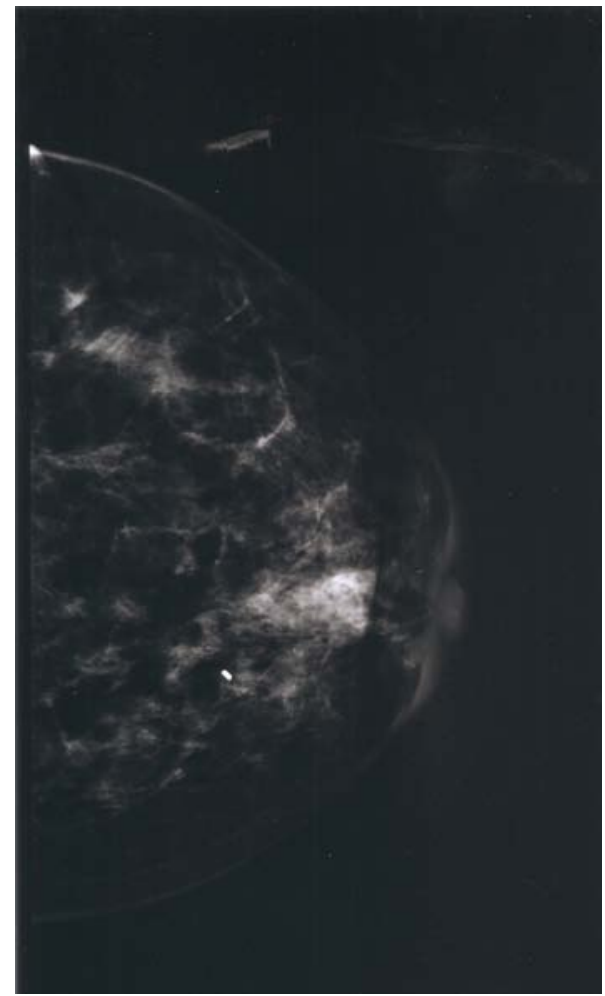

Figure 3. Mammogram craniocaudal view following core needle biopsy showing retroareolar granulomatous changes with periareolar skin thickening and nipple retraction. Biopsy clip seen on image.

ago. Her past medical history was significant for conversion to a positive tuberculin skin test, which was never treated. Serial chest radiographs were benign and showed no indication of current or prior pulmonary TB infection. There was no history of cough, hemoptysis, fevers, chills, night sweats or unexplained weight loss.

A preliminary diagnosis of cellulitic mastitis was made, and the patient was treated conservatively with antibiotics.

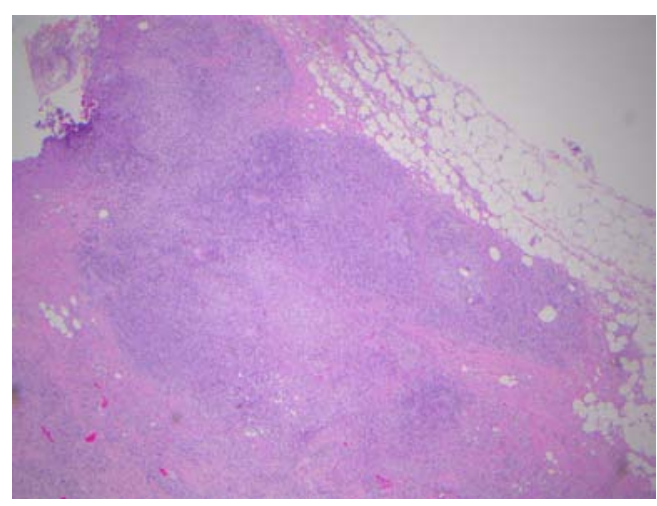

Figure 4. Low power $(\times 10)$ H\&E photomicrograph showing discrete granulomas. 


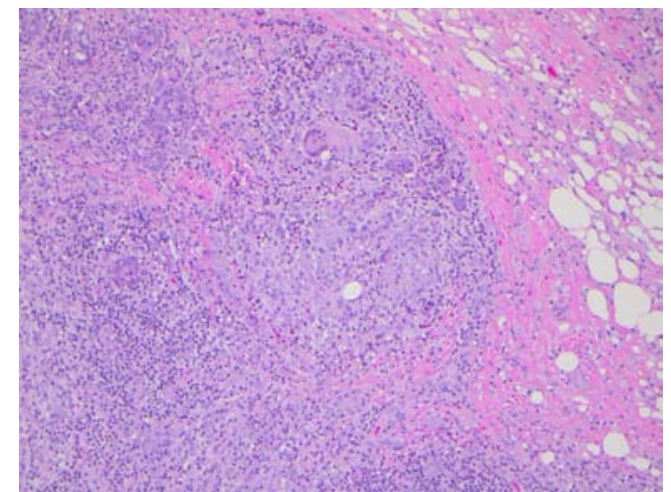

Figure 5. Photomicrograph H\&E specimen showing granulomatous inflammation manifested by lymphocytes, histiocytes and foreign body giant cells $(\times 40)$.

The pain and erythema resolved, but the induration persisted. The patient returned 1 month later with a new area of induration accompanied by overlying erythema, warmth and tenderness. At this time the patient was sent for further evaluation with imaging.

Mammography revealed findings suspicious of a tumor in the subareolar region with generalized skin thickening and nipple retraction. These findings are a significant change from the previous mammogram performed 7 months prior, which was normal. Ultrasound indicated two solid complex masses with irregular margins. The clinical history and imaging studies were highly suggestive of malignancy and indicated biopsy. The diagnostic hypotheses at this time included inflammatory breast cancer, infectious process or granulomatous mastitis.

A core needle biopsy was preformed. No organisms were seen on Gram stain but indicated 2+ white blood cells. Culture grew moderate diphtheroids. The mass was surgically incised and drained. Cytology revealed inflammation with predominantly neutrophils, lymphocytes, histiocytes, and foreign body giant cells in a background of acellular debris, fibrin and red blood cells. Cytology was negative for malignant cells. Surgical pathology indicated acute and chronic non-specific inflammation with granulation tissue and panniculitis. Surgery led to improvement in the pain and swelling, but the induration did not fully resolve.

Over the course of the next year, the patient presented with two more recurrences of left breast masses that were treated conservatively with antibiotics, which amounted to no significant improvement in her condition. The new masses were subsequently evaluated by mammography and ultrasound, and were again found to be suspicious for malignancy. Repeat ultrasound-guided core needle biopsies revealed benign pathology, and no further intervention was taken.

Two and a half years after initial presentation, the patient returned complaining of engorgement and erythema of the contralateral breast. The patient was treated conservatively with antibiotics without resolution of the induration. A new area of induration manifested and ultrasound revealed cystic rim enhancing foci. The patient was sent for MRI-guided core needle biopsy. MRI showed diffuse enhancement of the breast parenchyma with solid and cystic changes (Fig. 1, 2). Post-biopsy mammography revealed periareolar skin thickening with absence of positive findings in the area of palpable concern (Fig. 3). Core biopsy revealed non-specific granulomatous inflammation. The patient underwent incisional biopsy, and surgical pathology revealed noncaseating epithelioid granulomatous mastitis with chronic and acute inflammation, fibrosis, inflamed granulation tissue, fat necrosis and foreign body giant cell reaction (Fig. 4, 5). Microbiology and cultures were negative. The patient presented with recurrent abscesses twice over the next 2 months, which were treated with incision and drainage and oral antibiotics. Pathology and microbiology were consistent with previous findings.

The patient was referred to infectious disease and treated with 9 months of isoniazid and pyridoxine to reduce her risk of reactivation TB. The patient did not develop any more breast abscesses and remained asymptomatic.

\section{Discussion}

IGM is a rare and benign inflammatory disease of the breast of unknown etiology [1]. The disease is accepted to be idiopathic after excluding all other causes of granulomatous disease [9]. IGM is a diagnostic dilemma. The clinical findings of IGM are non-specific and can initially mimic other conditions, specifically inflammatory breast cancer, infection and other causes of granulomatous disease. When the presentation suggests infectious mastitis, trials of different antibiotics are frequently used. When there is no response to antibiotics, other etiologies should be suspected. It therefore requires a high degree of suspicion early in the course of disease to make a proper diagnosis of IGM. Definitive diagnosis requires incisional biopsy and histological examination.

In patients who are PPD positive, tuberculous mastitis must be carefully excluded. Breast TB should be suspected when there is poor response to non-TB antibiotics. Chest radiographs are useful in ruling out pulmonary TB; however, there are documented cases of tuberculous mastitis occurring in the absence of lung disease [12]. Histological diagnosis is therefore necessary to distinguish IGM from tuberculous mastitis by demonstrating granulomatous inflammation without caseous necrosis.

Treatment of IGM is challenging as there is no consensus in the literature. Initial treatment approaches vary widely and may include surgical or nonsurgical methods. IGM is generally a self-limiting condition and in uncomplicated cases can be safely observed without treatment $[9,13]$. Current practice indicates that in cases that require treatment, the 
initial approach should be non-operative and begin with a course of corticosteroids. In more persistent cases, immunosuppressives like methotrexate or azathioprine may be used. The addition of immunosuppressive agents allows for reduction in steroid dose. Surgical management should be considered as a last resort, especially in resilient or recurrent cases which have failed medical management or in cases that are complicated by abscess formation [14]. Surgery in cases of IGM can be challenging, requiring extensive resection to obtain disease-free margins, and even then, recurrence of IGM is high. Extensive scarring and fistula formation can occur, resulting in unsatisfactory cosmetic results [5]. Medical treatment with corticosteroids prior to surgery significantly decreases the inflammation, which allows for more conservative surgery and reduces the risk of recurrence $[5,15]$.

Treating IGM patients with latent TB infection presents a unique set of challenges. As steroids increase the risk of reactivation $\mathrm{TB}$, other treatment modalities should be employed. Little is previously documented about the effectiveness of pain management in patients with granulomatous mastitis. In patients with latent TB or others who cannot tolerate corticosteroid treatment, treatment should be primarily symptomatic with the aim of providing adequate pain relief. Adequate pain management may reduce the frequency of unnecessary antibiotic use and surgical palliation, and result in better long-term cosmetic outcomes.

\section{Acknowledgement}

The authors thank Dr. Shahida Ahmed of Department of Veterans Affairs, East Orange, NJ for preparation of pathology slides, and Dr. Basil Hubbi of Rutgers New Jersey Medical School, Newark, NJ for assistance with radiographic interpretation.

\section{Competing Interests}

We wish to confirm that there are no known conflicts of interest associated with this publication.

\section{Grant Support}

There has been no significant financial support for this work that could have influenced its outcome.

\section{Author Contributions}

LM reviewed the case notes and prepared the manuscript. GT edited and approved the final manuscript. This work was performed in Department of General Surgery, U.S. Depart- ment of Veterans Affairs, 385 Tremont Ave. (112), East Orange, NJ 07018, USA.

\section{Abbreviations}

IGM: idiopathic granulomatous mastitis; TB: tuberculosis

\section{References}

1. Kessler E, Wolloch Y. Granulomatous mastitis: a lesion clinically simulating carcinoma. Am J Clin Pathol. 1972;58(6):642-646.

2. Erhan Y, Veral A, Kara E, Ozdemir N, Kapkac M, Ozdedeli E, Yilmaz R, et al. A clinicopthologic study of a rare clinical entity mimicking breast carcinoma: idiopathic granulomatous mastitis. Breast. 2000;9(1):52-56.

3. Paviour S, Musaad S, Roberts S, Taylor G, Taylor S, Shore K, Lang S, et al. Corynebacterium species isolated from patients with mastitis. Clin Infect Dis. 2002;35(11):1434-1440.

4. Ocal K, Dag A, Turkmenoglu O, Kara T, Seyit H, Konca K. Granulomatous mastitis: clinical, pathological features, and management. Breast J. 2010;16(2):176-182.

5. Konan A, Kalyoncu U, Dogan I, Kilic YA, Karakoc D, Akdogan A, Kiraz S, et al. Combined long-term steroid and immunosuppressive treatment regimen in granulomatous mastitis. Breast Care (Basel). 2012;7(4):297301.

6. Seo HR, Na KY, Yim HE, Kim TH, Kang DK, Oh KK, Kang SY, et al. Differential diagnosis in idiopathic granulomatous mastitis and tuberculous mastitis. J Breast Cancer. 2012;15(1):111-118.

7. Pistolese CA, Di Trapano R, Girardi V, Costanzo E, Di Poce I, Simonetti G. An unusual case of bilateral granulomatous mastitis. Case Rep Radiol. 2013;2013:694697.

8. Lee JH, Oh KK, Kim EK, Kwack KS, Jung WH, Lee HK. Radiologic and clinical features of idiopathic granulomatous lobular mastitis mimicking advanced breast cancer. Yonsei Med J. 2006;47(1):78-84.

9. Patel RA, Strickland P, Sankara IR, Pinkston G, Many W, Jr., Rodriguez M. Idiopathic granulomatous mastitis: case reports and review of literature. J Gen Intern Med. 2010;25(3):270-273.

10. Imoto S, Kitaya T, Kodama T, Hasebe T, Mukai K. Idiopathic granulomatous mastitis: case report and review of the literature. Jpn J Clin Oncol. 1997;27(4):274-277.

11. Tse GM, Poon CS, Law BK, Pang LM, Chu WC, Ma TK. Fine needle aspiration cytology of granulomatous mastitis. J Clin Pathol. 2003;56(7):519-521.

12. Sriram K, Moffatt D, Stapledon R. Tuberculosis infection of the breast mistaken for granulomatous mastitis: a case report. Cases J. 2008;1(1):273. 
13. Erozgen F, Ersoy YE, Akaydin M, Memmi N, Celik AS, Celebi F, Guzey D, et al. Corticosteroid treatment and timing of surgery in idiopathic granulomatous mastitis confusing with breast carcinoma. Breast Cancer Res Treat. 2010;123(2):447-452.

14. Mazlan L, Suhaimi SN, Jasmin SJ, Latar NH, Adzman S, Muhammad R. Breast carcinoma occurring from chronic granulomatous mastitis. Malays J Med Sci. 2012;19(2):82-85.

15. Gurleyik G, Aktekin A, Aker F, Karagulle H, Saglamc A. Medical and surgical treatment of idiopathic granulomatous lobular mastitis: a benign inflammatory disease mimicking invasive carcinoma. J Breast Cancer. 2012;15(1):119-123. 